\title{
A RDWT and Block-SVD based Dual Watermarking Scheme for Digital Images
}

\author{
Sachin Gaur \\ Dept. of Electronics and Communication Engineering \\ Motilal Nehru National Institute of Technology \\ Allahabad, India
}

\author{
Vinay Kumar Srivastava \\ Dept. of Electronics and Communication Engineering \\ Motilal Nehru National Institute of Technology \\ Allahabad, India
}

\begin{abstract}
In the modern era, digital image watermarking is a successful method to protect the multimedia digital data for example copyright protection, content verification, rightful ownership identification, tamper detection etc. In this paper for improving the robustness and security, a Dual watermarking approach using Redundant Discrete Wavelet Transform (RDWT), block based singular value decomposition (SVD) and Arnold transform is presented. There are two gray scale watermarks, one is Prime watermark and other is Arnold scrambled Second watermark. Second watermark is embedded into the RDWT transformed Prime watermark in all sub bands to get the processed watermark image. After that transformed gray scale cover image is partitioned into non-overlapping blocks for embedding the processed watermark image by modifying the SVD coefficients of each block to obtain the resultant watermarked image. Now a reverse algorithm is developed to takeout the Prime and Second watermark from noisy image. Analysis and experimental outcomes show that the presented method is more robust against numerous image processing attacks and perform better as compared to previously introduced schemes related to presented work.
\end{abstract}

Keywords-Digital image watermarking; Redundant Discrete wavelet transform; Singular value decomposition; Arnold transform; NCC and PSNR

\section{INTRODUCTION}

Due to volatile revolution in the communication digital multimedia, internet and computer technology, the transportation of multimedia digital information/data over the internet are not safe and also suffers by unlawful operation such as unauthorized copying, duplication, editing, modification, replication, tampering and alterations by the inducers. So there is strong need for protection the digital content for ownership verification, copyright protection, content identification and temper detection etc. Now, it is a challenging task but watermarking is an important tool, which solve all these intellectual property rights problems. Watermarking is the process by which, the secret digital data information can hide into the digital multimedia (Audio, Image and Video) without any visible changing in the cover digital information and only true and real owner can extract the secret information. The concept of watermarking can also be used in various applications such as digital multimedia security, certification, Broadcast Monitoring, medical application, finger printing, data hiding etc. [1-3]. The elementary requirement of watermarking is imperceptibility, Robustness, Security. Robustness means that the watermarking procedure should be curbed beside several image processing attacks such as filtering, histogram manipulation, noise addition and geometric attacks such as translation, scaling, rotation, cropping etc. Imperceptible means sameness between original image data and watermarked image data. Security means resistance against malicious attacks.[3] For embedding the secret digital information in to the digital media is scaled by factor called scaling factor, if scale factor is high then more distortion in the image but the robustness is increased and vice versa. Watermarking techniques can be divided as spatial domain and frequency domain techniques. Spatial domain has high data capacity, less robust and transform domain techniques such as Discrete wavelet transform (DWT) Discrete Fourier transform (DFT), Discrete cosine transform (DCT), Singular value decomposition (SVD) are more robust and good imperceptibility [4-7]. The method of changing the location of picture pixel by way of matrix transforms to get an image whose visible effect is ailment known as scrambling. There are numerous scrambling methods of the image such as Hilbert fractal curve, Fibonacci-Q transform, Affine transform, Arnold transform [8-11].The hiding information embedding into the singular values of the source image data in SVD [12-15]. The RDWT based watermarking has the properties shift invariant directionality and overcome the DWT problem [16-19].Dual watermarking procedure in which two watermarks are embedded into the cover image in different ways for enhancing the robustness, imperceptibility and security [20-29].

The rest of the paper organized as in segment II literature survey, SVD, RDWT and Arnold transform are briefly described. Then in segment III embedding and extracting process are explained. The outcomes and the comparative analysis of our approach are shown in segment IV, and the conclusion of this paper is described in segment $\mathrm{V}$.

\section{LiteratURE SURVEY}

This section briefly explains the previous works on block based dual watermarking. Lin et al. [20] suggested a dual watermarking procedure using just noticeable distance method for invisible and visible watermark. Lee and Lin [21] presented a dual watermarking method for temper detection in which two replicas of watermark embedded in to each block of the image and improve the security and robustness. Hu et al. [22] offered a dual watermarking procedure for gray scale and binary watermark image and also improve the security and robustness. Dhanalakshmi and Thaiyalnayaki [23] suggested a DWT-SVD and chorus encryption based dual watermarking scheme but the 
robustness and imperceptibility results are not satisfactory. Amini et al. [24] proposed a DWT, block by block SVD dual watermarking scheme using principal component analysis. In this scheme embedded a binary watermark in best sub band on the basis of intensity variance of each block and also improve the robustness and security. Xiao et al. [25] presents a DWT based blind, dual watermarking method for color images. This scheme is used for invisible and fragile watermark for copyright protection and image authentication respectively. But the tamper localization is not satisfactory. Mohanty et al. [26] proposed a combination of visible and invisible block based dual watermarking scheme and embedding of watermark is done by finding the mean and variance of every block. Navas et al. [27] presented a DWT block based scheme and improve the imperceptibility as well as robustness. Lu and Liao [28] suggested a dual watermarking method for robust and fragile watermarks but tamper localization is not satisfactory. Ghazy et al. [12] proposed a block based SVD techniques, this scheme has very low embedding capacity. Bhatnagar et al. [29] provide a DWT-SVD base dual watermarking scheme in which a gray scale secondary watermark is embedded in the primary watermark and this primary watermark image is embedded in the host image but from the result analysis it is found that for some image processing attacks, the primary and secondary watermark are seriously degrade. In our presented scheme, a dual RDWT block-SVD based watermarking has high capacity and little degradation due the properties of RDWT and SVD and also enhances the robustness and imperceptibility.

\section{A. SVD}

Singular value decomposition(SVD) is a mathematical tool to dissolve any $n \times n$ matrix $A$ in to three matrices $U, D$ and $V$ as $A=U D V^{T}$ where $U$ and $V$ are left singular and right singular matrices which are orthogonal and $D=\operatorname{diag}\left(\alpha_{i}\right)$ is a diagonal matrix, where $\alpha_{i}, i=1,2,3 \ldots . n$ are the singular values and satisfies $\alpha_{1}>\alpha_{2}>\alpha_{3}>\cdots \cdots>\alpha_{n}$. The matrix $U$ and $V$ identify the geometric information and also singular matrix S contains the intensity information of the image [12]. The SVD matrix both may be square or rectangular. It can be factorized as

$$
\begin{aligned}
A & =U D V^{T} \\
& =\left[u_{1}, u_{2}, \ldots u_{n}\right] \times\left(\begin{array}{cccc}
\alpha_{1} & 0 & \ldots & 0 \\
0 & \alpha_{2} & \ldots & 0 \\
\vdots & 0 & \ddots & 0 \\
0 & 0 & \ldots & \alpha_{n}
\end{array}\right) \times\left[v_{1}, v_{2}, \ldots v_{n}\right] \\
& =\sum_{i=1}^{r} \alpha_{i} u_{i} v_{i}
\end{aligned}
$$

Where, 'r' represents the rank of matrix $A$. The SVD has good constancy property that means the minor deviation of singular values do not disturb the visual characteristics of the digital information[13,14] It is also used in various application such as noise reduction, image watermarking, noise and hiding of the image etc.

\section{B. Arnold Transform}

Vladimir Arnold proposed cat mapping called Arnold transform in the research of Ergodic theory. Arnold transform is one the method to achieve encryption algorithm in image transform domain $[8,10]$. It is 2D chaotic map, when it applied on digital images, then the original pixel position become irregular and the image seem to be imperceptible or distorted image (scrambled image). Arnold transform can be described as:

$\left|\begin{array}{l}a^{\prime} \\ b^{\prime}\end{array}\right|=\left|\begin{array}{ll}1 & 1 \\ 1 & 2\end{array}\right|\left|\begin{array}{l}a \\ b\end{array}\right|(\bmod M)$

The inverse Arnold transform can be defined as

$\left|\begin{array}{l}a \\ b\end{array}\right|=\left|\begin{array}{rr}2 & -1 \\ -1 & 1\end{array}\right|\left|\begin{array}{l}a^{\prime} \\ b^{\prime}\end{array}\right|(\bmod M)$

The value of $a, b=\{0,1,2 \ldots \ldots \ldots \ldots . . M-1\}$

Where $(a, b)$ are the original image pixel coordinates and $\left(a^{\prime} b^{\prime}\right)$ indicates the coordinates of the image pixels after applying Arnold transform. The size of square matrix is $M$. Each pixel of the image is passed over by Arnold transform equation and after several iterative calculations the distorted image (scrambled) is obtained. The image is recovered after certain number of permutation due to periodicity of Arnold transforms [11].

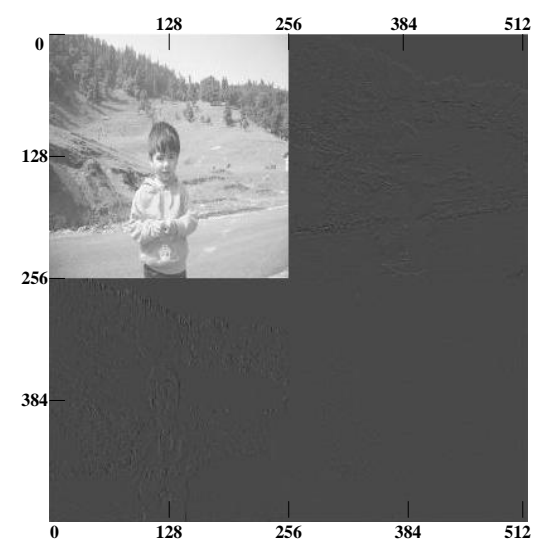

Fig. 1. 1-level Haar DWT (Rudra Image size is $512 \times 512$ and decompose each sub-band size is $256 \times 256$ )

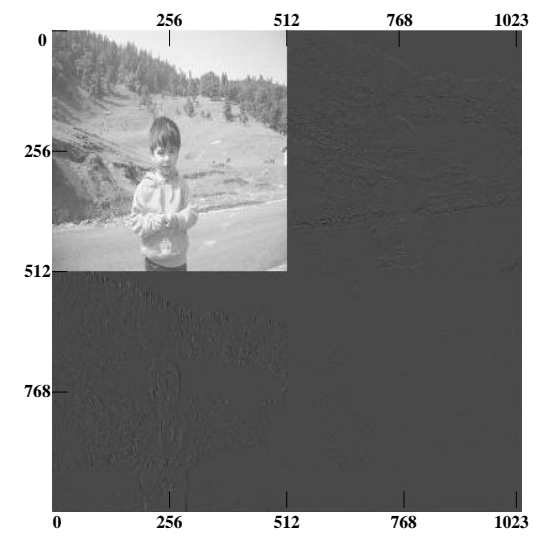

Fig. 2. -level Haar RDWT (Rudra Image size is $512 \times 512$ and decompose each sub-band size is $512 \times 512$ )

\section{C. $R D W T$}

In the image processing application, for transforming the image in to transform domain from spatial domain, generally DWT is mostly using common transform because it has spatiofrequency localization property. It affords sufficient statistics to investigate and synthesize the data with a substantial decrease within the computational complexity. The down sampling approach in DWT attains shift variant even for a slight shift in 
the entire image. Due to shift variant a major changes occur in the image wavelet coefficients as well as slight shifts occur in the input image $[16,17]$. Due to this, there is an incorrect extraction of watermark image and cover image data. This is main shortcoming of DWT based watermarking. To overcome the problem of DWT, the RDWT techniques have been proposed because it has directionality, shift invariant properties $[18,19]$. From Fig.1and2, at the same decomposition level the size of sub band is decrease in DWT but in RDWT is same as the host image.

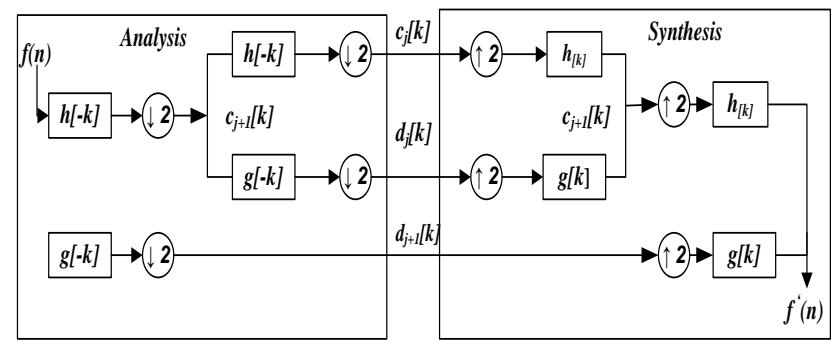

Fig. 3. Analysis and Synthesis filter banks of ID DWT

Fig. 3 and Fig.4 represents the 1D DWT and 1D RDWT and their inverse transform respectively. Where $f(n)$ and $f^{\prime}(n)$ shows the 1D input, reconstruct the signal. $h[-k]$ and $g[-k]$ represents analysis filters, $h[k]$ and $g[k]$ shows synthesis low pass, high pass filters respectively. $c_{j}$ and $d_{j}$ are low and high band output coefficients at $j$ level. Difference between DWT and RDWT are demonstrated by the equation (4-12) and Fig.3 and 4.

DWT analysis and synthesis equations can be expressed as

Analysis equation

$c_{j}[k]=\left(c_{j+1}[k] * h[-k]\right) \downarrow 2$

$d_{j}[k]=c_{j+1}[k] * g[-k] \downarrow 2$

If $y[n]=x[n] \downarrow 2$

Then $y[n]=x[2 n]$

Synthesis equations given as

$c_{j+1}[k]=\left(\left(c_{j}[k] \uparrow 2\right) * h[k]+\left(d_{j}[k] \uparrow 2\right) * g[k]\right)$

If $y[n]=x[n] \uparrow 2$, then

DWT can be described by

$y[n]=\left\{\begin{array}{cc}x\left[\frac{n}{2}\right] & , n \text { is even } \\ 0 & , n \text { is odd }\end{array}\right.$

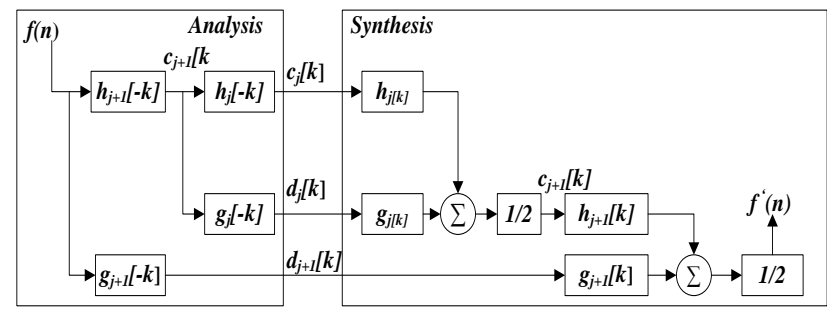

Fig. 4. Analysis and Synthesis filter banks of I D RDWT

RDWT analysis and synthesis equations can be expressed
Analysis equations

$c_{j}[k]=\left(c_{j+1}[k] * h_{j}[-k]\right.$

$d_{j}[k]=\left(c_{j+1}[k] * g_{j}[-k]\right.$

Synthesis

$c_{j+1}[k]=\frac{1}{2}\left(c_{j}[k] * h_{k}[k]+d_{j}[k] * g_{i}[k]\right)$

Where * means convolution and $\downarrow 2$ means down sampling and $\uparrow 2$ means up sampling at each level of iteration in DWT. Size of each sub band decrease due to down sampling and increase in levels of decomposition. RDWT discard down sampling as well as up sampling of coefficients. At every stage the output coefficient two times that of the input. Due to shift invariant, directionality, spatio-frequency localization property RDWT based digital image processing techniques is more robust than DWT based techniques.

\section{PROPOSED SCHEME}

The proposed scheme embeds the dual watermarking method for enhancing the robustness and security. In this scheme there are two invisible watermarks, one is Prime watermark ' $P_{R}$ ' and other is Second watermark ' $S$ ' both are same size $(128 \times 128)$ gray scale images and ${ }^{\prime} H^{\prime}$, is a gray scale cover image of size $512 \times 512$. First the Arnold scrambled Second watermark embedded into Prime watermark and get the processed watermark image and this processed watermark image is considered as a watermark image for the cover image. The Embedding and extracting steps are given below.

\section{A. Watermark Embedding}

\section{a) Second Watermark Embedding Steps}

For embedding of Second watermark ' $S$ ' into the Prime watermark ${ }^{\prime} P_{R}{ }^{\prime}$ is given as.

1) Second watermark ' $S$ ' is scrambled by the Arnold transform and get Arnold scrambled watermark image $S_{N A}$.

2) Apply SVD Process on this scrambled watermark image $S_{N A}$ such as.

$$
S_{N A}=I_{S} S W_{S} V_{S}^{T}
$$

3) Perform one level RDWT transform on the Prime watermark $\left(P_{R}\right)$ to divide it into 4-sub bands and get the $P_{R j}$ image, where $j=\{L L, L H, H L$ and $H H$ sub bands.

4) Apply SVD Process on Prime watermark of all sub bands such as.

$$
P_{R j}=U W_{P j} S W_{P j} V W_{P j}^{T}
$$

5) Now modified the singular values of the Prime watermark by adding the singular values of the Second watermark in all sub bands such as.

$$
S W_{P j}+\alpha S W_{S}=S W_{j}^{*}
$$

6) Now find the new RDWT coefficient matrix by applying inverse SVD transform for all sub bands.

$$
P_{R j}^{*}=U W_{P j} S W_{j}^{*} V W_{P j}^{T}
$$

7) Now apply inverse RDWT and modified the coefficient of all sub bands and get the processed watermark image as.

$$
P W_{R}^{*}=R D W T^{-1}
$$




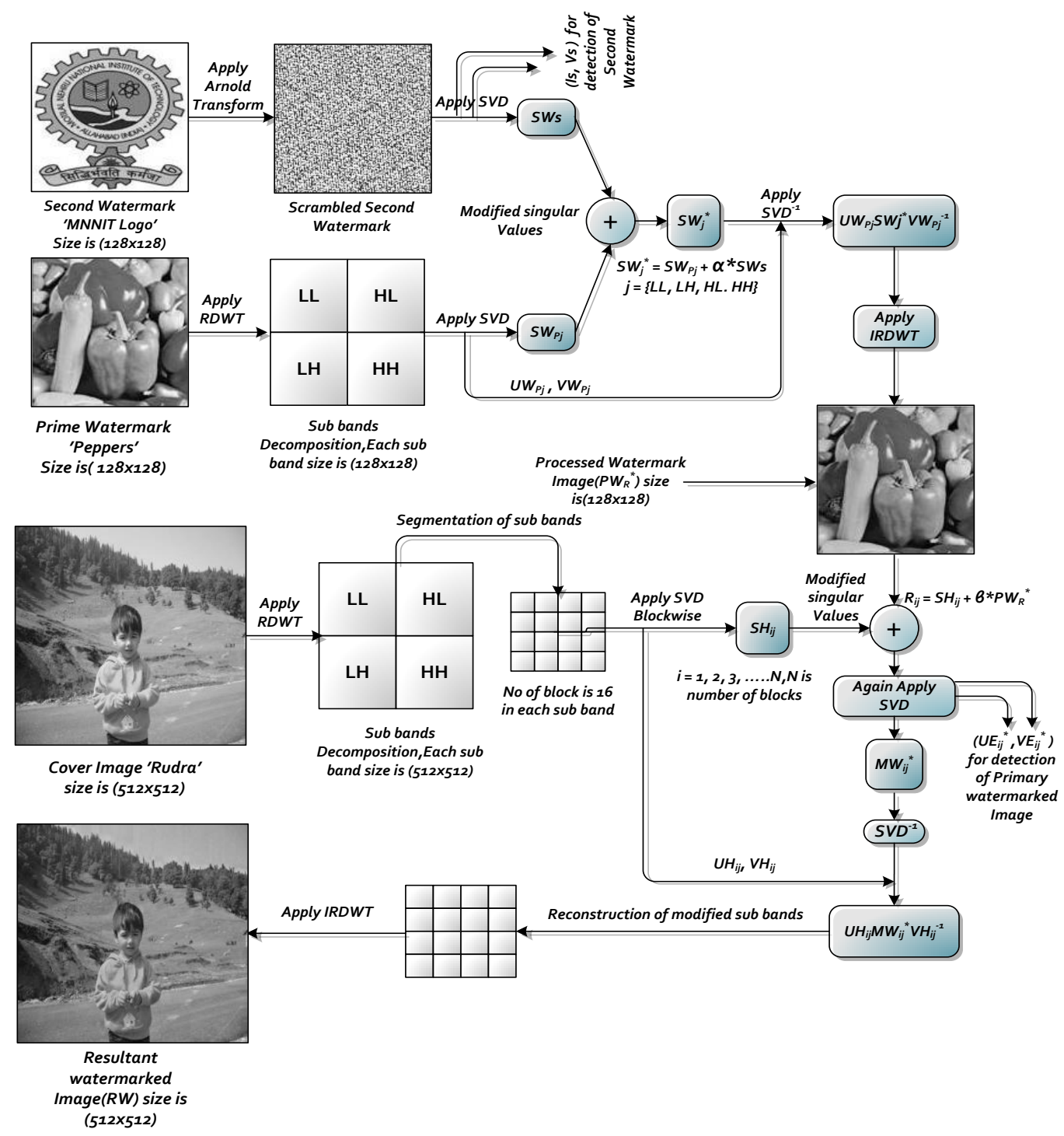

Fig. 5. Watermark Embedding Process

\section{b) Embedding of Prime watermark image}

Now the processed watermark image $P W_{R}^{*}$ use as watermark for the cover image and the embedding steps are given bellow.

1) One-level RDWT transform is carried out on the cover image for obtaining four sub bands (LL, LH, HL and HH).

2) Divide the approximation and details part into non overlapping blocks.

3) An SVD process is applied on every block sub bands and gets the $S H_{i j}$ matrix of singular values. Where $i$ $=1,2,3 \ldots . . N, N$ are the no. of blocks and $j=\{L L, L H, H L$, and HH\} sub bands.

$C_{i j}=U H_{i j} S H_{i j} V H_{i j}^{T}$
4) Now modified cover image singular values, directly by adding the processed watermark image $P W_{R}^{*}$ such as. $S H_{i j}+\beta P W_{R}^{*}=R_{i j}$

5) Again SVD process is applied on the modified matrix $R_{i j}$ such as.

$R_{i j}^{*}=U E_{i j}^{*} M W_{i j}^{*} V E_{i j}^{* T}$

6) Now perform the operation such as.

$L_{i j}=U H_{i j} M W_{i j}^{*} V H_{i j}^{*}$

7) Now at the end apply IRDWT and reconstruct the all watermark image in each bands and get the resultant watermarked image $R W$. 


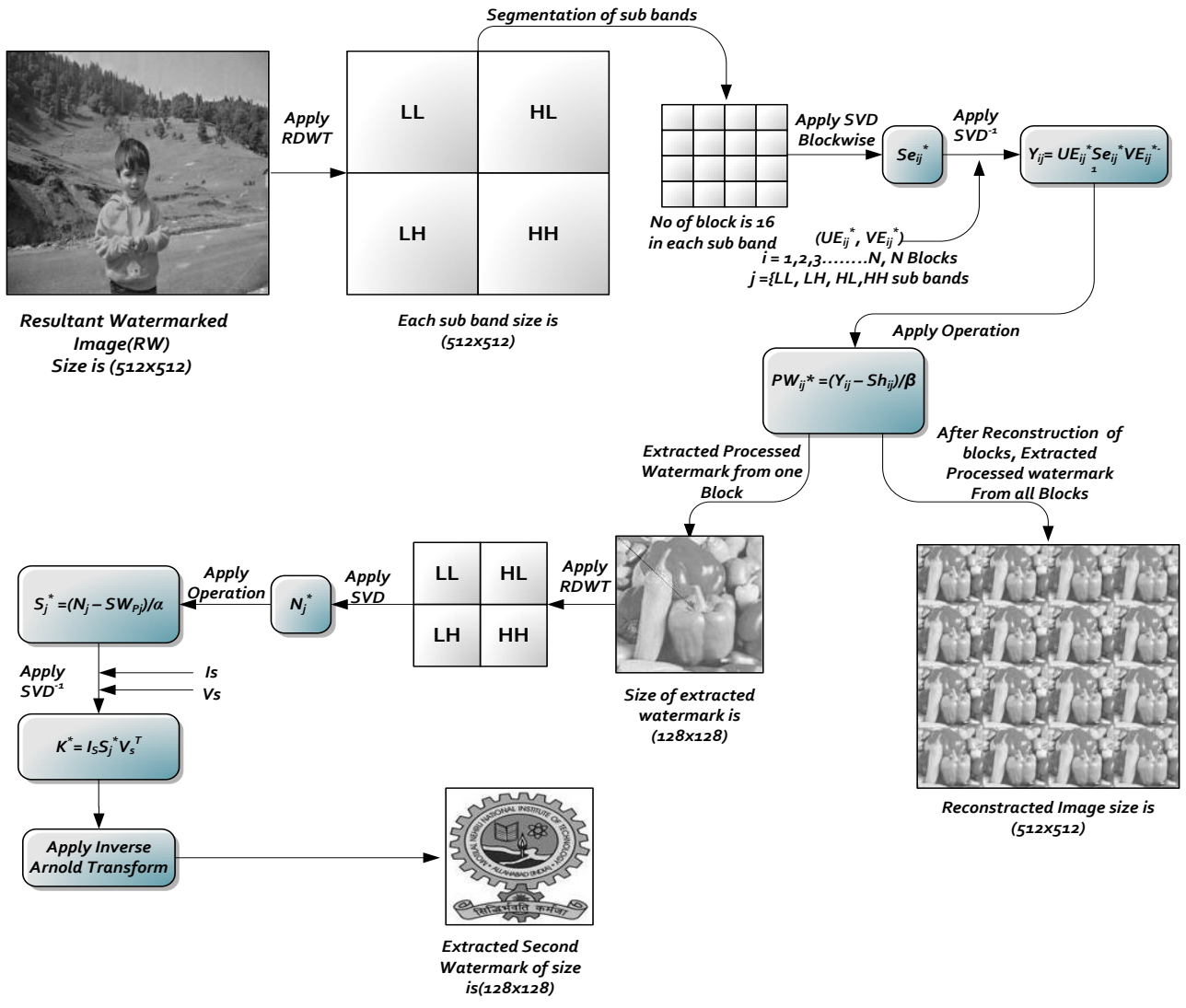

Fig. 6. Watermark Extraction Process

\section{B. Watermark Extraction}

For extraction the inverse process of embedding is applied, first extract the processed watermark image from the cover image which is known as watermark for the cover image and then extract the second watermark from this image. The extraction steps are given below.

\section{a) Extraction of Prime Watermark}

1) First apply RDWT on noisy corollary watermarked image $R W$ and four sub bands (LL, LH, HL and $\mathrm{HH}$ ) are obtained.

2) After that segmented these sub bands in to nonoverlapping blocks.

3) Now SVD transform is applied on each block of all sub bands such as.

$E W_{i j}=E_{i j} S e_{i j}^{*} H_{i j}^{T}$

4) Now applying inverse SVD transform such as.

$Y_{i j}=U E_{i j}^{*} S e_{i j}^{*} V E_{i j}^{*}$

5) Now applying this operation

$P W_{i j}^{*}=\left(\frac{Y_{i j}-S H_{i j}}{\beta}\right)$
6) Now rearrange all extracted processed watermark in each block of all sub bands and get the combine image.

b) Extractions of Second Watermark Image

Now finally takeout the Second watermark from processed watermark image. The extraction steps are given as:

1) Processed watermark image which has highest correlation coefficient is decomposed into four sub bands using RDWT.

2) Perform SVD process on each sub bands such as $D=G_{j} N_{j}^{*} B_{j}^{T}$

3) Apply this operation

$S_{j}^{*}=\frac{N_{j}^{*}-S W_{P j}}{\alpha}$

4) Now obtain the extracted scramble Second watermark such as.

$\breve{K}=I_{S} S_{j}^{*} V_{s}^{T}$

5) At the end apply inverse Arnold transform and get the Second watermark image $\bar{K}^{*}$.

$\widetilde{K^{*}}=\operatorname{IAT}(\widetilde{K})$ 


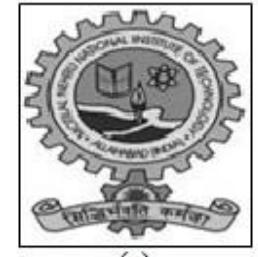

(a)

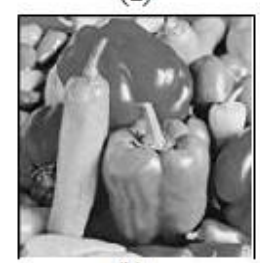

(b)

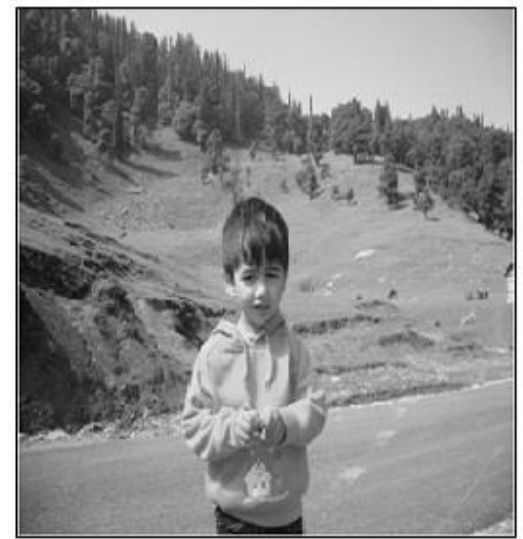

(c)
Fig. 7. Input Images (a) Second Watermark 'MNNIT Logo' (b) Prime Watermark 'Peppers'(c) Cover Image 'Rudra'

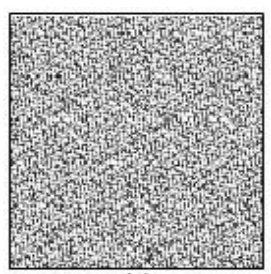

(a)

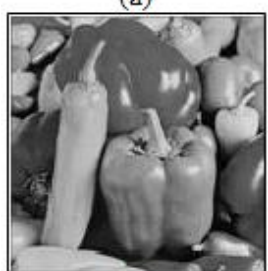

(b)

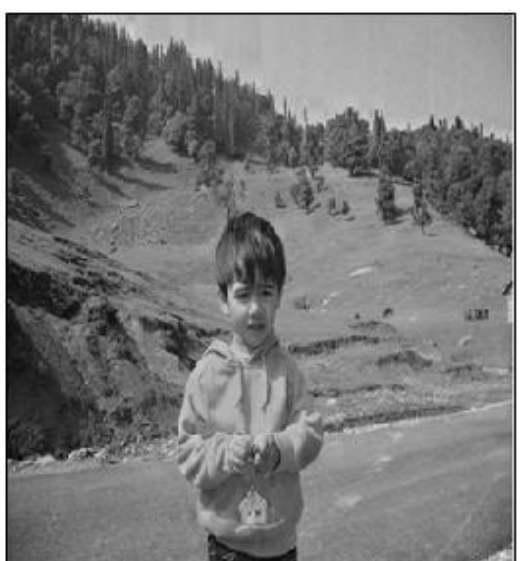

(c)
Fig. 8. Images after embedding (a) Scrambled Second Watermark Image 'MNNIT Logo' (b) Processed Watermark Image 'Peppers' (PSNR=43.381db $\mathrm{NCC}=0.9886$ ) (c) Resultant watermarked Image 'Rudra' (PSNR=49.564db, $\mathrm{NCC}=0.9991$ )

\section{EXPERIMENTAL RESULTS}

In this segment experiments are executed to evaluate the performance of proposed method by using the MATLAB 13a. In our experiments there are three gray scale images have been taken, one is prime watermark 'peppers' of size $128 \times 128$ and other is second watermark 'MNNIT Logo' of same size and third one is cover image 'Rudra' of size 512x512 respectively. Now decompose the cover image into one level RDWT by using 'Haar' mother wavelet filter bank and segmented in to non-overlapping blocks and then embedded the processed watermark image 64 times into the cover image. For extraction of Second watermark, those processed watermark images have been taken whose correlation coefficient is highest. Different block size can be use but this size has less complexity. Fig.7 shows the source images (a) Second watermark (MNNIT Logo) (b) Prime watermark (peppers) (c) cover image (Rudra) and Fig.8 shows the (a) scrambled Second watermark (b)
Processed watermark and (c) resultant watermarked image. For preserving the imperceptibility and robustness, here two scaling factor one is 0.05 for embedding the Second watermark into the Prime watermark and other (0.02) for embedding the processed watermark image in the cover image have been taken. The PSNR(peak signal to noise ratio) is calculated to analysis the imperceptibility of the image. The acceptable value of PSNR is $30 \mathrm{db}$.

The PSNR can be calculated by the given formula as.

$P S N R=10 \log _{10}\left[\frac{\max (x(i, j))^{2}}{M S E}\right]$

MSE (Mean square error between host image $x$ and watermarked image $y$ can be calculated as

$M S E=\frac{1}{m \times n} \sum_{i=1}^{M} \sum_{j=1}^{N}[x(i, j)-y(i, j)]^{2}$

The robustness can be calculate by finding the normalize correlation coefficient (NCC) by given formula.

$$
=\frac{N C(w, \bar{w})}{\sqrt{\sum_{i=1}^{M} \sum_{j=1}^{N}\left[w(i, j)-\mu_{w}\right]^{2}} \sqrt{\sum_{i=1}^{M} \sum_{j=1}^{N}\left[\bar{w}(i, j)-\mu_{\bar{w}}\right]^{2}}}
$$

Where $N$ and $M$ indicates the no of pixel of watermark. $w, \bar{w}$ are real and extracted watermark. $\mu_{w}, \mu_{\bar{w}}$, represent the mean values of real and extracted watermark respectively. The value of NCC should be in the range between -1 and 1 . If the value of NCC closes to +1 then the extracted watermark is very much correlated. If it's far close to -1 , the extracted watermark is also very much correlated, however it happens to be a negative images. And if it's value is equal to 0 , the extracted watermark is absolutely uncorrelated, generally the NC acceptable value is 0.75 or above. To examine the robustness and imperceptibility of propose method, different image processing attacks for example Gaussian noise, salt and pepper noise, speckle noise, rotation, resizing, compression, cropping, median filtering are applied on the resultant

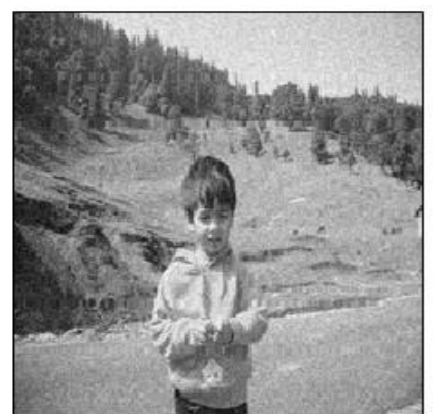

(a)

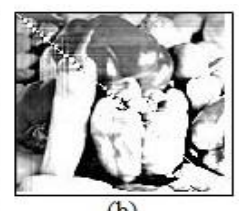

(b)

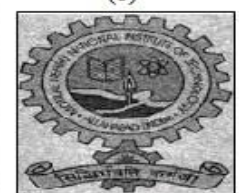

(c)
Fig. 9. Gaussian Noise (0.5) (a) Attacked Cover image (b) Extracted Prime Watermark (c) Extracted Second Watermark 

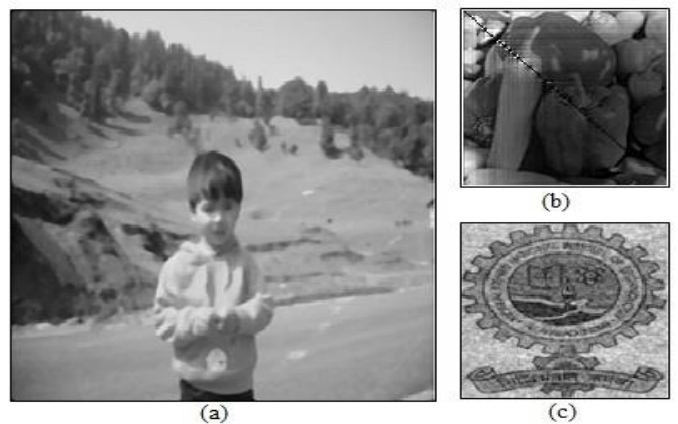

(b)

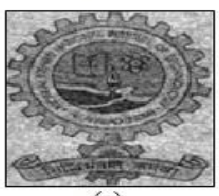

(c)

Fig. 10. Median filtering (7×7) (a) Attacked Cover image (b) Extracted Prime Watermark (c) Extracted Second Watermark

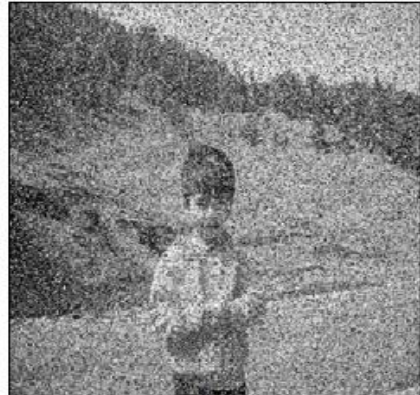

(a)
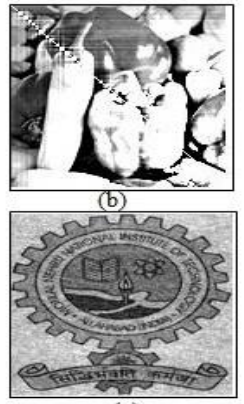

(c)
Fig. 11. Salt \& Pepper Noise (0.4) (a) Attacked Cover image (b) Extracted prime watermark (c) Extracted Second Watermark
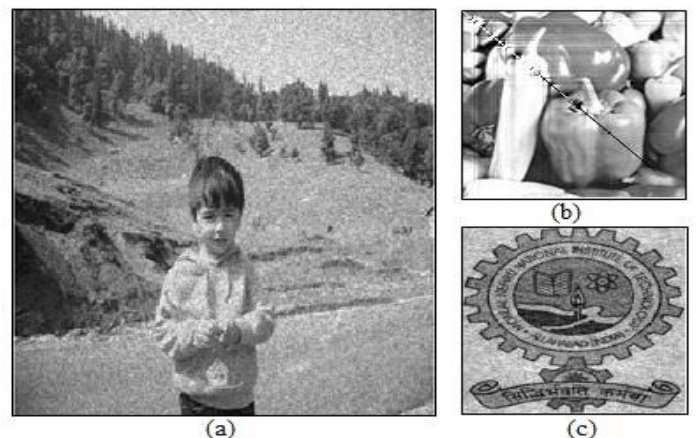

(c)

Fig. 12. Speckle Noise (density0.4) (a) Attacked Cover image (b) Extracted Prime Watermark (c) Extracted Second Watermark

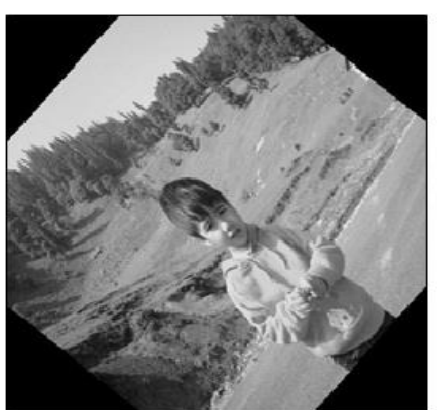

(a)

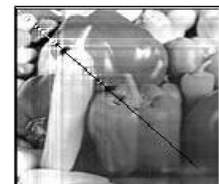

(b)

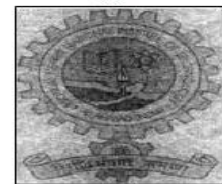

(c)
Fig. 13. Rotation $\left(50^{\circ}\right)$ (a) Attacked Cover image (b) Extracted Prime Watermark (c) Extracted Second Watermark

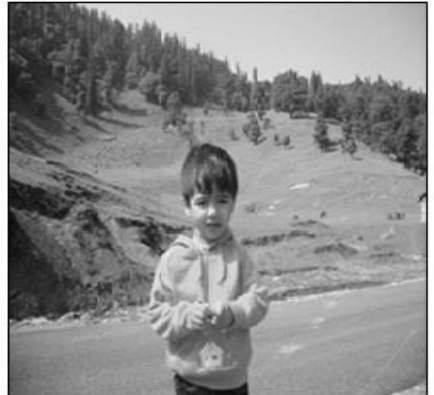

(a)
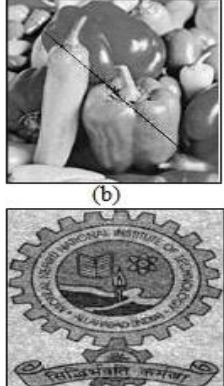

(c)
Fig. 14. Compression (a) Attacked Cover image (b) Extracted Prime Watermark (c) Extracted Second Watermark

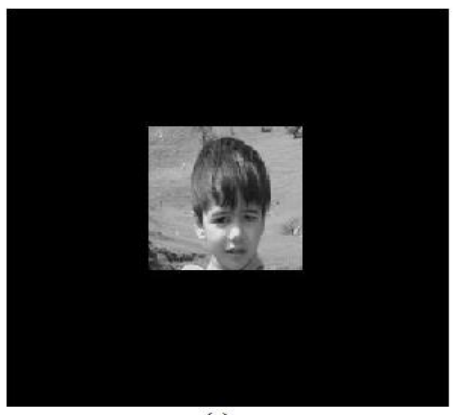

(a)

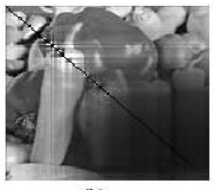

(b)

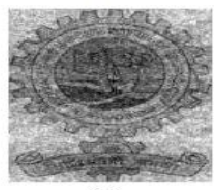

(c)
Fig. 15. Cropping (30\%) (a) Attacked Cover image (b) Extracted Prime watermark (c) Extracted Second Watermark

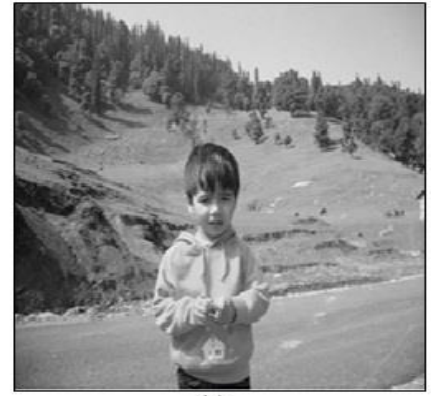

(a)

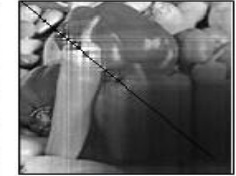

(b)

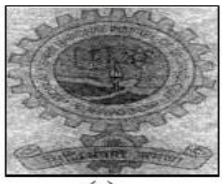

Fig. 16. Resizing (512-256-512) (a) Noisy Cover image (b) Extracted Prime watermark (c) Extracted Second watermark

watermarked image and see the correlation between extracted watermarks and original image by finding the correlation coefficient using equation (31) in Table 1and 5. The PSNR (peak signal to noise ratio) of processed watermark image and resultant watermarked image are $(43.381 \mathrm{db}, 49.564 \mathrm{db})$ respectively can be calculated by using the equation(29),30. The outcomes of extracted watermark after applying attacks gaussian noise (mean $=0$,var $=0.5$ ), pepper and salt noise $(0.4)$, speckle noise $(0.4)$, median filtering $(7 \times 7)$, rotation $\left(50^{\circ}\right)$, cropping(30\%), compression(75:1), resizing (512-256-512) are shown in Fig. 9-16. and also have been depicted in the Table 1. Table 1 presents the minimum correlation coefficient value after applying attacks which is greater than (0.5) so the presented method is accaptable. 
TABLE. I. CORRELATION COEFFICIENT VALUE OF PRIME AND SECOND WATERMARK IMAGE AFTER APPLYING ATTACKS IN LL SUB-BAND

\begin{tabular}{|l|l|l|}
\hline \multirow{2}{*}{ Attacks } & NCC \\
\cline { 2 - 3 } & $\begin{array}{l}\text { Prime } \\
\text { Watermark }\end{array}$ & $\begin{array}{l}\text { Second } \\
\text { Watermark }\end{array}$ \\
\hline (a) Gaussian Noise(M=0,V=0.5) & 0.6377 & 0.5365 \\
\hline (b) Salt \& Pepper noise(0.4) & 0.5954 & 0.5131 \\
\hline (c) Speckle noise(0.4) & 0.5998 & 0.5117 \\
\hline (d) Median filtering(7×7) & 0.5676 & 0.4989 \\
\hline (e) Rotation(50 $\left.{ }^{0}\right)$ & 0.6083 & 0.4991 \\
\hline (f) Cropping (30\%) & 0.6187 & 0.5091 \\
\hline (g) Resizing(512-256-512) & 0.6365 & 0.5783 \\
\hline (h) Compression & 0.6963 & 0.5998 \\
\hline
\end{tabular}

Now also check this proposed scheme for different standard images like Lena, Penguin, Baboon, and Boat and also find out the numerical analysis values. After embedding the watermark the values of correlation coefficient and PSNR (peak signal to noise ratio), for these images as well as extracted watermark are shown in Table 2 and 3. The highest correlation coefficient of Prime and Second extracted watermark after applying attacks shown in the Table.5 After analysis of received outcomes, conclude that the provided method is wealthy in terms of robustness, security and imperceptibility.

TABLE. II. NCC AND PSNR VALUE AFTER EMBEDDING THE PROCESSED WATERMARK IN DIFFERENT IMAGES

\begin{tabular}{|l|c|l|l|}
\hline \multicolumn{2}{|l|}{ Images } & NCC & PSNR \\
\hline Baboon & Lena & 0.9894 & 54.973 \\
\hline Bird & S & 0.9991 & 57.184 \\
\hline Boat & 0.9881 & 53.142 \\
\hline
\end{tabular}

TABLE. III. NCC AND PSNR VALUE AFTER EXTRACTING THE PRIME WATERMARK IN DIFFERENT IMAGES

\begin{tabular}{|l|l|l|}
\hline \multirow{2}{*}{ Images } & \multicolumn{2}{|l|}{ Prime Watermark } \\
\cline { 2 - 3 } & NCC & PSNR \\
\hline Baboon & 0.9773 & 58.991 \\
\hline Lena & 0.9792 & 60.347 \\
\hline Bird & 0.9669 & 59.144 \\
\hline Boat & 0.9632 & 60.891 \\
\hline
\end{tabular}

TABLE. IV. NCC AND PSNR VALUE AFTER EXTRACTING THE SECOND WATERMARK IN DIFFERENT IMAGES

\begin{tabular}{|l|l|l|}
\hline \multirow{2}{*}{ Images } & \multicolumn{2}{|l|}{ Second Watermark } \\
\cline { 2 - 3 } & NCC & PSNR \\
\hline Baboon & 0.9665 & 48.898 \\
\hline Lena & 0.9689 & 50.387 \\
\hline Bird & 0.9614 & 45.557 \\
\hline Boat & 0.9558 & 47.149 \\
\hline
\end{tabular}

\section{A. Comparative Study}

In this segment, compare the results of the presented method with Bhatnagar et al. [29] shown in Table 5.Bhatgnar et al. [29] proposed a DWT-SVD based dual watermarking method in which a gray scale secondary watermark is embedded in the primary watermark and this primary watermark image is embedded in the host image by using zigzag sequencing but from the result analysis it is found that for some image processing attacks, the primary and secondary watermark are seriously degrade.

TABLE. V. COMPARISON OF HIGHEST CORRELATION COEFFICIENT VALUE OF PRIME AND SECOND WATERMARK IMAGE AFTER APPLYING ATTACKS IN LL SUB-BAND BETWEEN PROPOSED SCHEME AND BHATNAGAR ET AL. [29] ,''MEANS ATTACKS ARE NOT APPLY

\begin{tabular}{|l|l|l|l|l|}
\hline \multirow{2}{*}{ Attacks } & \multicolumn{3}{|l|}{ NCC comparison } \\
\cline { 2 - 5 } & \multicolumn{2}{|l|}{ Bhatnagar et al.[29] } & \multicolumn{2}{l|}{ Proosed Scheme } \\
\cline { 2 - 5 } & $\begin{array}{l}\text { Prime } \\
\text { Water- } \\
\text { mark }\end{array}$ & $\begin{array}{l}\text { Second } \\
\text { Water } \\
\text { mark }\end{array}$ & $\begin{array}{l}\text { Prime } \\
\text { Water- } \\
\text { mark }\end{array}$ & $\begin{array}{l}\text { Second } \\
\text { Water } \\
\text { mark }\end{array}$ \\
\hline $\begin{array}{l}\text { (a) Gaussian } \\
\text { Noise(M=0,V=0.5) }\end{array}$ & 0.4728 & 0.3527 & 0.7832 & 0.6598 \\
\hline $\begin{array}{l}\text { (b) Salt \& Pepper } \\
\text { noise(0.4) }\end{array}$ & - & - & 0.6945 & 0.5937 \\
\hline (c) Speckle noise(0.4) & - & - & 0.6783 & 0.6214 \\
\hline (d) Median filtering(7×7) & 0.5432 & 0.3314 & 0.6685 & 0.5963 \\
\hline (e) Rotation(50 $)$ & 0.5627 & 0.2822 & 0.7538 & 0.6018 \\
\hline (f) Cropping (30\%) & 0.5366 & 0.3249 & 0.7128 & 0.5382 \\
\hline (g) Resizing(512-256-512) & 0.7776 & 0.2863 & 0.8193 & 0.6185 \\
\hline (h) Compression & 0.9887 & 0.7393 & 0.8189 & 0.7841 \\
\hline
\end{tabular}

In our proposed method, a $128 \times 128$ Arnold scrambled gray second watermark image is embedded in to the prime watermark image of same size and get processed watermark image, this processed watermark image is embedded in to the RDWT transformed cover image of size $512 \times 512$. RDWT has shift invariant, directionality property due to this, RDWT based watermarking method is more robust than DWT based watermarking. The comparison between our method and Bhatnagar scheme for Prime and Second watermark are also shown by comparative chart in Fig.17. By seeing the NCC values in Table 1,5, Fig.9-16 and comparative chart, we can analyze that the presented techniques is more robust against distinct image processing attacks and geometric attacks and have high embedding capacity, more imperceptible and robust than previously proposed techniques.

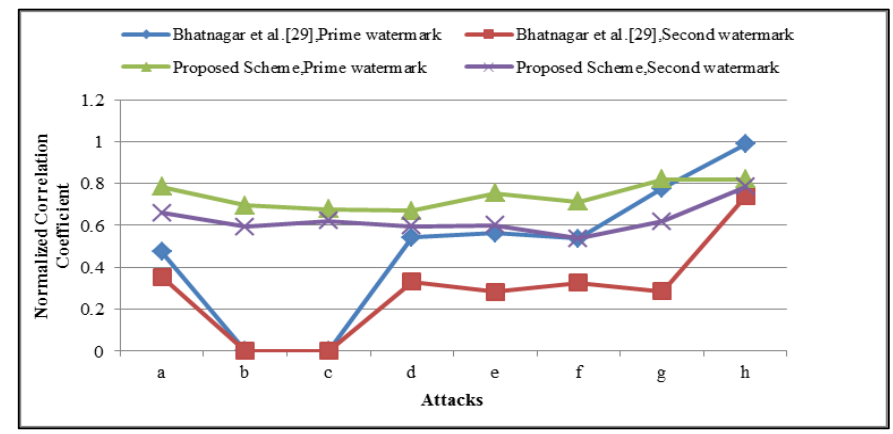

Fig. 17. Comparative Chart between Bhatnagar et al.[29] and proposed scheme

\section{CONCLUSION}

This paper presents a dual image watermarking method based on RDWT and block-SVD, to embed an Arnold scrambled Second watermark image. The watermarking process is carried out by modifying the singular values of prime watermark to obtain a processed watermark image, 
which is then embedded by modifying the singular values of each block with all RDWT sub bands of the gray scale cover image. For extraction the left and right singular matrices are used at the receiver end.This scheme has the high embedding capacity and a little degradation in the image due to the RDWT-SVD properties and scrambled watermark by Arnold transform to enhance its privacy. The considerable contribution of this presented scheme is for copyright protection and authentication of the digital images. The future scope of this scheme is an optimization method can also be used for different images to make it adaptive. Experimental results show that it sustain the noise and various image processing, geometric attacks and also improve the performance in terms of imperceptibility, capacity robustness, and security.

\section{REFERENCES}

[1] K. Arai, "Secure Copier Which Allows Reuse Copied Documents with Sorting Capability in Accordance with Document Types," International Journal of Advanced Research in Artificial Intelligence, vol. 2, no. 9, pp. 14-18, 2013.

[2] Matt L. Miller, I. J. Cox, J.-P. M. G. Linnartz, and T. Kalker, “A Review of of Watermarking Principles and Practices," Digital Signal Processing for Multimedia Systems, pp. 461-485, 1999.

[3] E. Hussein and M. A. Belal, "Digital Watermarking Techniques , Applications and Attacks Applied to Digital Media: A Survey," International Journal of Engineering Research \& Technology, vol. 1, no. 7, pp. 1-8, 2012.

[4] K. Arai and C. Ragmad, "Image Retrieval Method Utilizing Texture Information Derived from Discrete Wavelet Transformation Together with Color Information," International Journal of Advanced Research in Artificial Intelligence, vol. 5, no. 10, pp. 1-6, 2016.

[5] C.-C. Lai and C.-C. Tsai, "Digital Image Watermarking Using Discrete Wavelet Transform and Singular Value Decomposition," IEEE Transactions on Instrumentation and Measurement., vol. 59, no. 11, pp. 3060-3063, 2010.

[6] P. Bao and X. Ma, "Transactions Letters," IEEE Transactions on Circuits System for Video Technology, vol. 15, no. 1, pp. 96-102, 2005.

[7] K. Arai and C. Ragmad, "Wavelet Based Image Retrieval Method," International Journal of Advanced Computer Science and Applications, vol. 3, no. 4, pp. 6-11, 2012.

[8] G. Chen, Y. Mao, and C. K. Chui, "A symmetric image encryption scheme based on 3D chaotic cat maps," Chaos, Solitons and Fractals, vol. 21, no. 3, pp. 749-761, 2004.

[9] D. Qi, J. Zou, and X. Han, "A new class of scrambling transformation and its application in the image information covering," Science in China (Series E) Technological Science, vol. 43, no. 3, pp. 304-312, 2000.

[10] R. Ye, "A novel image scrambling and watermarking scheme based on orbits of Arnold transform," Proceedings of the Pacific-Asia Conference on Circuits, Communications and System (PACCS), no. 1, pp. 485-488, 2009.

[11] L. Lu, X. Sun, and L. Cai, "A Robust Image Watermarking based on DCT by Arnold Transform and Spread Spectrum,"3rd International Conference on Advanced Computer Theory and Engineering (ICA CTE), pp. 198-201, 2010.

[12] R. A. Ghazy, A. M. Abbas, N. Al-Zubi, E. S. Hassan, N. a. El-Fishawy, M. M. Hadhoud, M. I. Dessouky, E.-S. M. El-Rabaie, S. A. Alshebeili, and F. E. Abd El-Samie, "Block-based SVD image watermarking in spatial and transform domains," International Journal of Electronics, vol. 102, no. 7, pp. 1091-1113, 2015.

[13] C.-C. Chang, C.-C. Lin, and Y.-S. Hu, "An SVD Oriented Watermark Embedding Scheme with High Qualities for the Restored Images," International Journal of Innovative Computing, Information and Control, vol. 3, no. 3, pp. 609-620, 2007.

[14] R. Liu, T. Tan, and S. Member, "An SVD-Based Watermarking Scheme for Protecting," IEEE Transaction on Multimedia, vol. 4, no. 1, pp. 121128, 2002.

[15] E. Ganic and A. M. Eskicioglu, "Robust embedding of visual watermarks using discrete wavelet transform and singular value decomposition," J. Electron. Imaging, vol. 14, no. 4, pp. 1-12, 2005.

[16] N. M. Makbol and B. E. Khoo, "Robust blind image watermarking scheme based on Redundant Discrete Wavelet Transform and Singular Value Decomposition," Journal of Electronic Imaging (AEÜ), vol. 67, pp. 102-112, 2013.

[17] T. Duy, Z. Nakao, and Y. Chen, "Robust multi-logo watermarking by RDWT and ICA," Signal Processing, vol. 86, pp. 2981-2993, 2006.

[18] S. Lagzian, M. Soryani, and M. Fathy, "Robust watermarking scheme based on RDWT-SVD: Embedding data in all subbands," International Symposium on Artificial Intelligence and Signal Processing (AISP), pp. $48-52,2011$.

[19] J. Guo and H. Prasetyo, "Security analyses of the watermarking scheme based on redundant discrete wavelet transform and singular value decomposition,"International Journal of Electronics and Communication vol. 68, no. 9, pp. 816-834, 2014.

[20] P. Y. Lin, J. S. Lee, and C. C. Chang, "Dual digital watermarking for internet media based on hybrid strategies," IEEE Transaction on Circuits System Video Technology, vol. 19, no. 8, pp. 1169-1177, 2009.

[21] T. Y. Lee and S. D. Lin, "Dual watermark for image tamper detection and recovery," Pattern Recognit., vol. 41, no. 11, pp. 3497-3506, 2008.

[22] M. C. Hu, D. C. Lou, and M. C. Chang, "Dual-wrapped digital watermarking scheme for image copyright protection," Computers and Security, vol. 26, no. 4, pp. 319-330, 2007.

[23] R. Dhanalakshmi and K. Thaiyalnayaki, "Dual Watermarking Scheme with Encryption," International Journal of Computer Science and Information Securit, vol. 7, no. 1, pp. 248-253, 2010.

[24] M. Amini, K. Yaghmaie, and H. Sadreazami, "A new scheme for dual watermarking using DWT-PCA technique," International Conference on Imaging Theory and Applications, pp. 43-46., 2010.

[25] X. Liu, C. Lin, and S. Yuan, "Blind dual watermarking for color images" authentication and copyright protection," IEEE Transactions on Circuits System for Video Technology, vol. 1051-8215, no. c, pp. 1-9, 2016.

[26] S. P. Mohanty, K. R. Ramakrishnan, and M. Kankanhalli, "A Dual Watermarking Technique for Images," ACM Multimedia, vol. 99, no. Part 2, pp. 49-51, 1999.

[27] K. A. Navas, M. C. Ajay, M. Lekshmi, T. S. Archana and M. Sasikumar, "DWT-DCT-SVD based watermarking," 3rd International Conference on Communication Systems Software and Middleware and Workshops (COMSWARE), pp. 271-274, 2008.

[28] C. S. Lu and H. Y. M. Liao, "Multipurpose watermarking for image authentication and protection," IEEE Transaction on Image Processing, vol. 10, no. 10, pp. 1579-1592, 2001.

[29] G. Bhatnagar, B. Raman, and K. Swaminathan, "DWT-SVD based Dual Watermarking Scheme," First International Conference on the Applications of Digital Information and Web Technologies, pp. 526-531, 2008. 